\title{
Angular and seasonal variation of spectral surface reflectance ratios: Implications for the remote sensing of aerosol over land.
}

\author{
L.A. Remer \\ Laboratory for Atmospheres (code 913), NASA/Goddard Space Flight Center, \\ Greenbelt, Maryland, 20771 (remer@climate.gsfc.nasa.gov) \\ A.E. Wald \\ Science Applications International Corporation, Chantilly, Virginia 20151 \\ (andy@stillwater.saic.com) \\ Y.J.Kaufman \\ Laboratory for Atmospheres (code 913), NASA/Goddard Space Flight Center, \\ Greenbelt, Maryland, 20771 (kaufman@climate.gsfc.nasa.gov)
}

submitted to

IEEE Transactions on Geoscience and Remote Sensing

August 30, 1999 


\begin{abstract}
We obtain valuable information on the angular and seasonal variability of surface reflectance using a hand-held spectrometer from a light aircraft. The data is used to test a procedure that allows us to estimate visible surface reflectance from the longer wavelength $2.1 \mu \mathrm{m}$ channel (mid-IR). Estimating or avoiding surface reflectance in the visible is a vital first step in most algorithms that retrieve aerosol optical thickness over land targets. The data indicate that specular reflection found when viewing targets from the forward direction can severely corrupt the relationships between the visible and $2.1 \mu \mathrm{m}$ reflectance that were derived from nadir data. There is a month by month variation in the ratios between the visible and the mid-IR, weakly correlated to the Normalized Difference Vegetation Index (NDVI). If specular reflection is not avoided, the errors resulting from estimating surface reflectance from the mid-IR exceed the acceptable limit of $\Delta \rho \sim 0.01$ in roughly $40 \%$ of the cases, using the current algorithm. This is reduced to $25 \%$ of the cases if specular reflection is avoided. An alternative method that uses path radiance rather than explicitly estimating visible surface reflectance results in similar errors. The two methods have different strengths and weaknesses that require further study.
\end{abstract}

\title{
1.0 Introduction
}

When viewed from space, the striking difference between land and ocean surfaces is the uniformity of the ocean and the large spatial variability of the land. Land surface reflectance is dependent on the type of vegetation and the type of soil, as well as the relative fraction of each (Huete and Tucker, 1990). Surface reflectance varies as the vegetation progresses through its growing cycle. It also varies due to seasonal changes in soil moisture and hydrological 
surface characteristics such as snow cover. Furthermore, surface reflectance varies by the geometry of the relative viewing and illumination angles, and the geometrical variation is dependent on vegetation types (Deering, et al., 1999; Tsay, et al., 1998).

The extreme variability of reflectance from the land surface hinders the remote sensing of aerosol over land. One exception is the contrast reduction technique, in which the effect of aerosol in reducing the apparent variability of the surface reflectance is used to determine the change in the aerosol optical thickness (Tanré, et al., 1988). While there have been successful operational satellite retrievals of aerosol over the comparatively uniform ocean surface (Husar, et al., 1997), attempts at an operational algorithm over land has eluded us. A few specific cases using Advanced Very High Resolution Radiometer (AVHRR) (Vermote, et al., 1992) have been successful. Likewise aerosol indices derived from Total Ozone Mapping Spectrometer (TOMS) and POLarization and Directionality of Earth Reflectances (POLDER) provide qualitative or experimental aerosol information over land surfaces (Herman, et al., 1997; Herman, et al., 1997). Still, we have been missing a quantitative, operational aerosol product over land.

With the launch of the NASA Terra satellite carrying the MODerate resolution Imaging Spectroradiometer (MODIS), among other instruments, a new era in remote sensing will begin. One of the fundamental advances of the new sensor will be the regular retrieval of a quantitative aerosol product over land surfaces (Kaufman, et al., 1997a; Chu, et al., 1998). MODIS has this capability due to the inclusion of mid-infrared channels, especially the $2.1 \mu \mathrm{m}$ band. Most aerosol types (e.g. smoke or urban pollution) are transparent to the radiation in the mid-IR (Kaufman and Remer, 1994). Even dust, though 
having particles of the size of the $2.1 \mu \mathrm{m}$ wavelength has only a small effect on the apparent surface reflectance of deserts as seen from space (Kaufman, et al., 1999). The reflectance in the $2.1 \mu \mathrm{m}$ channel peers through the obstructing aerosol to characterize the surface reflectance. Hence, we can identify the dark surface pixels.

In previous work Kaufman, et al. (1997b) establish the relationships between the visible and mid-IR channels as

$$
\begin{aligned}
& \rho_{\text {red }}=0.50 \rho_{2.1} \\
& \rho_{\text {blue }}=0.25 \rho_{2.1}
\end{aligned}
$$

These relationships were derived from data collected mostly from the United States' east coast in July 1993 using high altitude spectral images, Airborne Visible-IR Imaging Spectrometer (AVIRIS) and Landsat Thematic Mapper (TM). Both sensors view the earth's surface from the nadir direction only. Kaufman, et al. (1997b) used observations of aerosol optical thickness measured by ground-based sunphotometers with the apparent reflectance measured by the high altitude sensors in a radiative transfer code to derive the surface reflectances that were used to formulate Equations (1). Equations (1) were tested using independent data collected by other means, including a subset of the data presented in this study. The complete aerosol remote sensing algorithm was tested in the Eastern United States and Brazil (Kaufman, et al., 1997a; Chu, et al., 1998). The results of the testing showed that the relationships in Equation (1) were adequate to estimate visible surface reflectance and derive aerosol optical thickness to within the specified theoretical error bounds. However, the testing was performed on a limited data base of mostly high altitude sensors, at nadir view and during one month in the seasonal growing season. 
In this study, we analyze data collected from a sensor aboard a low flying aircraft over Charles County Maryland $\left(38^{\circ} 30^{\prime} \mathrm{N}, 77^{\circ} 05^{\prime} \mathrm{W}\right)$. The low altitude eliminates the need to derive surface reflectance from high altitude apparent reflectance. The data are collected for off-nadir views, and the analysis emphasizes possible disruption to Equation (1) from angular effects. By repeating measurements in the same location over several months we also examine whether the relationships expressed in Equation (1) hold during the progression of a typical growing season in a temperate climatic zone.

\subsection{Experimental Design}

We collected spectra of the ground using a handheld Analytic Spectral Devices (ASD) spectrometer while flying over various surface targets in a Cessna at an altitude of $300 \mathrm{~m}$. The spectra span the range from $440 \mathrm{~nm}$ to $2500 \mathrm{~nm}$. The spectral resolution is $3 \mathrm{~nm}$ in the range $400-1000 \mathrm{~nm}$, and $10 \mathrm{~nm}$ for longer wavelengths. The field of view is $18^{\circ}$ full field, corresponding to a spot size of $\sim 100 \mathrm{~m}$ from a platform at $300 \mathrm{~m}$ above the ground. The instrument head was held by hand out of the window with a nominal view angle of $45^{\circ} \pm 10^{\circ}$. Five to ten spectra were taken of each target during an elapsed time of roughly 15 to 30 seconds. The targets were of homogeneous surface type, $600-1000 \mathrm{~m}$ wide. The data were checked for spurious incursions into the field of view, and improper spectra were discarded, then the remaining spectra were averaged for each target. We used a spectral square transmission function to calculate the reflectance in each MODIS band. In this study we compare the ratios between different MODIS bands. These

ratios were calculated from channels of a given spectrum. All channels were taken simultaneously, at the same view angle. 
The instrument was used in reflectance mode in which the radiance is normalized by baseline values taken of a white barium sulfate plate. White plate measurements were made immediately before boarding the aircraft. Flight duration was typically one hour, and flight days were chosen to correspond to days of low optical thickness and homogeneous sky (Table 1). In this way sky conditions remained unchanged during the flight. Atmospheric correction based on the $6 \mathrm{~s}$ radiative transfer code (Vermote, et al., 1997) is applied to the data to correct for the Rayleigh scattering in the 300 $\mathrm{m}$ air column beneath the plane. The correction affects only the $0.47 \mu \mathrm{m}$ channel, and then only slightly.

The five flights over a two year period (Table 1) span the natural vegetative seasonal cycle in the mid-Atlantic region. We revisited the same targets or similar targets on each flight and thus have a spectral record of the changing surface reflectance of surfaces ranging from deciduous forests to crop land to marshes and pasture. During the flights we noted the surface type of each target and the approximate viewing geometry, either forward, backward or perpendicular to the principal plane. On two flights (May 1996 and July 1997) the solar zenith angle was too small to cast sufficient shadows on the ground for us to make a determination of the approximate azimuth of our viewing geometry.

\subsection{Angular Dependence}

The ratios between the visible and mid-IR developed from mostly nadir measurements in Kaufman, et al. (1997b) are expected to hold better for the backward scattering direction than for the forward scattering direction. As the viewing angle of the target approaches specular reflection, the spectral signature of the reflectance lessens. This occurs because the specularly 
reflected sunlight originates at the interface between the air and the plant cuticular wax layer. The photons never enter the leaves, never interact with the liquid water or the pigments in the leaves, and therefore, are reflected off of the leaves with little spectral signature (Vanderbilt and De Venecia, 1988; Rondeaux and Vanderbilt, 1993). In a plant canopy of randomly oriented leaves, specular reflectance can occur at all viewing geometries. However, plant canopies are not necessarily randomly oriented and previous studies (Vanderbilt and De Venecia, 1988; Rondeaux and Vanderbilt, 1993) have shown specular reflection to have preferential orientation to specific viewing angles, especially in the forward scattering direction when the sun is low in the sky.

Figure 1 shows the relationships between the visible and mid-IR channels from flights in different months. The data are also given in Table 2. The slopes for the blue channel range from 0.21 to 0.41 , as contrasted with the mean value of Kaufman, et al. (1997b) of 0.25 . In the red channel the range is from 0.38 to 0.53 , as compared to the Kaufman, et al. (1997b) value of 0.50 . However, some flights exhibit a strong correlation between the visible and mid-IR (March and July), while other flights do not (April and October).

The low correlation during the flights of April and October is mainly due to a subset of points identified in Figure 2. These points represent targets that span the entire range of surface types found in the data set, including forest, corn stubble and short grass. No standing water was noted in any of these targets. The flights exhibiting poor correlation occurred during the spring or fall when the vegetation was not yet fully leafed out or was already beginning to fade into senescence. However, the March flight, in another spring month with even less leaf area exhibits a high correlation between mid-IR and visible reflectance. 
The commonality of the points causing the low correlation in the April and October flights is that each of those targets was viewed in the forward scattering direction. Figure 2 also shows the results of excluding all targets identified as being viewed from the forward scattering position. The results greatly increase the correlation in the April and October flights and bring the slopes of those lines closer to the expectations of Equation (1). Table 2 gives the ratios and correlation coefficients for each flight, with and without targets viewed from the forward scattering direction. On the other hand, removal of the forward scattering points in the March flight data does not change the statistics for that flight.

Physically we are seeing the effect of specular reflection from the leaf canopies. Like glint on water, specular reflection from a plant canopy causes the reflected radiation to lose its spectral signature (Vanderbilt and De Venecia, 1988; Rondeaux and Vanderbilt, 1993). The ratios between the visible and mid-IR approach 1.0. Note the solar zenith angles from Table 1. The April and October observations were made when the sun was lowest in the sky, even lower than the March flight. Rondeaux and Vanderbilt (1993) show that the specular component of the reflected light increases with increasing solar zenith angle, although there are some dependencies on canopy architecture. According to Rondeaux and Vanderbilt (1993), the specular reflectance of the March flight with solar zenith angle of $46^{\circ}$ would be less likely to dominate our measurements taken with view angle of $45^{\circ}$ than would the April and October flights with solar zenith angle of $51^{\circ}$.

Data collected in Israel on May 8, 1997 using an identical experimental procedure were collected when the solar zenith angle was $40^{\circ}$. According to Rondeau and Vanderbilt (1993), the specular component of the reflectance should be small. If so, then we would expect a strong correlation between the 
visible and mid-IR channels. Figure 3 shows the scatter plot between the visible and mid-IR reflectance for the May 8th data collected in Israel. The forward scattering points are highlighted in blue. As expected, the correlation is strong, and the forward scattering points do not behave differently from targets viewed from other directions.

Other angular measurements of plant canopies also point to the angular dependence of the ratio between visible and mid-IR surface reflectance. Tsay, et al. (1998) describe a set of measurements of different surface types using the Cloud Absorption Radiometer (CAR) in Brazil. The CAR permits the measurement of the full bidirectional reflectance of the surface at several different wavelengths. The CAR data show that ratio between the surface reflectance of two visible channels and the $2.2 \mu \mathrm{m}$ channel is dependent on viewing geometry, with the ratio increasing in the forward scattering direction (Tsay, et al., 1998; Gatebe et al, in preparation).

The results of the analysis of the off nadir data suggest specular reflection may introduce significant error to the MODIS aerosol retrieval algorithm. Therefore we shall explore the improvement in remote sensing of aerosol over the land if the immediate region around the specular reflection is avoided, somewhat similar to the MODIS procedure used over the oceans (Tanré, et al., 1997).

\subsection{Seasonal and Surface Cover Dependence}

The ratio between the visible and mid-IR surface reflectance exhibits a seasonal dependence. For both the blue and red channels the ratio decreases during the height of the growing season when the Normalized Difference Vegetation Index (NDVI) is at its peak. NDVI is defined as

$$
\mathrm{NDVI}=\left(\rho_{0.87}-\rho_{0.66}\right) /\left(\rho_{0.87}+\rho_{0.66}\right)
$$


where $\rho_{0.87}$ is the surface reflectance at $0.87 \mu \mathrm{m}$ and $\rho_{0.66}$ is the surface reflectance at $0.66 \mu \mathrm{m}$. Figure 4 shows the seasonal variation of the monthly mean values of the ratio for (1) all the targets, (2) all the targets except marshes, (3) only forest targets, and (4) all target types without forward scattering. Table 2 lists all ratios and all correlation coefficients.

The subset of "forest only" targets represent the targets of highest NDVI. As a group these highly vegetated surfaces dramatically decrease the $0.66 \mu \mathrm{m}$ ratio during the summer flights, while increasing the $0.47 \mu \mathrm{m}$ ratio during the October flight. In both cases the forest subset moves the ratio further from the expected values of 0.50 and 0.25 , respectively. The "forest only" subset represents the darkest targets with the lowest reflectance. Because the forests are so dark (Kaufman and Remer, 1994), even though the percentage error in estimating visible reflectances will be large, the absolute error of applying Equation 1 will be small.

The sensitivity to NDVI is shown in Figure 5 for the data from all flights combined. All forward scattering targets have been eliminated. Ratios decrease as NDVI increases, especially at $0.66 \mu \mathrm{m}$. However, the scatter in the relationships is large. Figure 5 suggests that knowledge of the target NDVI may be useful in modifying the coefficients of Equation 1 in some circumstances. For example, in regions of extremely dark, dense vegetation where NDVI exceeds 0.85, it may be preferable to decrease the coefficient at $0.66 \mu \mathrm{m}$. The primary difficulty will be obtaining an atmospherically corrected NDVI. Using clear sky composite values may be the answer. The suggested relationship between the ratios and the vegetation index in this data set needs to be further examined in similar data collected in other regions of the world before the global algorithm can be modified. 


\subsection{Errors in Estimating Surface Reflectance}

What are the errors in estimating visible surface reflectance using Equation (1) for this data set? This question must be posed in context of the intended application. The purpose of estimating surface reflectance using Equation (1) is to provide the basis for the remote sensing of aerosol over land. The remote sensing method is inherently a "dark target" method. Equation (1) is not indiscriminately applied to each and every pixel observed by the satellite. Instead there is an order of operation that was developed to minimize contamination and errors. The algorithm proceeds as follows:

- All pixels in a $10 \mathrm{~km}$ by $10 \mathrm{~km}$ grid box are grouped together for analysis. The MODIS $2.1 \mu \mathrm{m}$ channel has a $500 \mathrm{~m}$ spatial resolution. Therefore each analysis grouping includes up to 400 cloud free pixels.

- Only, dark pixels, those pixels with $\rho_{2.1}<\rho_{\text {cutoff }}$ are included in the analysis. Currently MODIS uses a cascade of cutoffs: $\rho_{\text {cutoff }}=0.05$, 0.10 and 0.15 . We shall test the last cutoff in this study.

- A filter is applied to the visible reflectance of remaining pixels. The brightest $60 \%$ as well as the darkest $10 \%$ measured in terms of the pixels' visible reflectance are discarded. We refer to this as the 10$40 \%$ filter. This filter is an operational check to eliminate clouds accidentally left unmasked, cloud shadows or other unexpected contamination in the procedure.

- Equation 1 is applied to each of the remaining pixels, visible reflectance is estimated and the aerosol optical thickness retrieved.

If there are less than 40 pixels that survive such a selection process, then no aerosol retrieval is attempted for that $10-\mathrm{km}$ by $10-\mathrm{km}$ square. 
We apply Equation 1 to our data set, flight by flight, for different combinations of constraints and selection processes. We include in this test a constraint on forward scattering. Forward scattering targets are removed at the time the 0.15 cutoff selects the dark targets, before the $10-40 \%$ filter is applied. In each month of our data we assume that the targets represent the different pixels encountered in a $10 \mathrm{~km}$ square. A major difference between our testing procedure in this study and actual situations to be encountered by MODIS is our lack of pixels. While MODIS analysis will include up to 400 pixels in cloud free conditions, we observed as little as 15 targets on one our flights. In actuality, each of our flights contains fewer targets than the minimum necessary for the algorithm to proceed. Nonetheless, these are the available data, and we proceed with the testing in order to see how imposing the different constraints affects the error in estimating visible reflectance.

Figure 6 illustrates the severity of imposing the different constraints. This figure shows the combined data set of all five flights. The targets remaining after the current constraints are imposed are represented by the combination of red and filled black points. Except for the darkest $10 \%$ of the targets, which do not pass the $10-40 \%$ filter, the surviving points are the darkest targets. The blue points are those targets viewed from the forward scattering direction. When these points are eliminated first, before imposing the current constraints, the surviving targets are reduced to the set of filled black points only.

Error is defined as $\rho_{\lambda}{ }^{\text {meas }}-\rho_{\lambda}{ }^{\text {calc }}$ where $\rho_{\lambda}{ }^{\text {meas }}$ is the observed reflectance at wavelength $\lambda$, and $\rho_{\lambda}{ }^{\text {calc }}$ is the reflectance calculated from Equation 1 at the same wavelength. Figure 7 shows and Table 3 lists the average error due to imposing different sets of constraints on our different data sets. Because each month contains so few targets compared to the 
anticipated 400 pixels from the MODIS analysis, we add a sixth data set in which we combine all months to create a data base of 125 pixels. The combined data base spanning the growing season is an artificial conglomerate and may introduce a higher degree of variability of land surface reflectance than would typically be available in a $10 \mathrm{~km}$ by $10 \mathrm{~km}$ square. The combined data set is labeled as "total" in Figure 7 and Table 3. The label "no constraints" refers to the application of Equation 1 for all the targets, all the angles, with no imposed dark target threshold and no 10-40\% filter. The label "dark targets" refers to imposing a cutoff of $\rho_{2.1}<0.15$ in order to include only the darkest targets. The label "current constraints" refer to those pixels that survive the $\rho_{2.1}<0.15$ cutoff and the $10-40 \%$ filter, but have no restriction on the view angle. Finally the category "current+no forw" is the most restrictive, and eliminates all forward scattering targets in addition to imposing the current constraints.

We must estimate the surface reflectance in the visible to within $\sim 0.01$ in order to retrieve optical thickness to within $\sim 0.10$. Imposing no constraints at all on the data introduces unacceptable errors in half the cases. Simply restricting the analysis to dark targets reduces the error in July, but not enough to bring it down to acceptable levels at $0.66 \mu \mathrm{m}$, and in April and October, "dark targets" actually increases the error. Passing the dark targets through the $10-40 \%$ filter improves the situation. The "current restraints" which include both the $\rho_{2.1}<0.15$ cutoff and the $10-40 \%$ filter on the visible reflectance results in $40 \%$ of the cases having unacceptable errors. However, once the forward scattering targets are removed from the "current restraints" category, only $25 \%$ of the cases introduce unacceptable levels of error. Part of this improvement is due to the elimination of all pixels from the April flight. 
Although the selection process reduces the error in estimating surface reflectance from the mid-IR, unacceptable error occurs in $25 \%$ of the cases. These cases are estimates in the red channel for the May and July flights and the combined data set of all sites. The combined data is dominated by the May/July data for the darkest targets. The lower panel of Figure 4 illustrates the reason for the error. The surviving targets designated by filled black points, all fall below the diagonal line representing Equation (1). These very dark, dense vegetated surfaces at the height of the growing season do not conform to the expected spectral ratio. The reason is unclear.

\subsection{Comparison with a Path Radiance Technique}

A different method for the remote sensing of aerosol over land that makes use of the $2.1 \mu \mathrm{m}$ channel has been proposed (Wen, et al.,1999). Similar to the ratio method, this new method assumes that the atmosphere is transparent to aerosols in the mid-IR so that after accounting for gaseous absorption, $\rho_{2.1}^{*}=\rho_{2.1}^{\mathrm{S}}$ where $\rho_{2.1}^{*}$ is the apparent reflectance at the top of the atmosphere and $\rho_{2.1}$ is the surface reflectance at $2.1 \mu \mathrm{m}$. We can express the apparent visible reflectance as

$$
\rho_{\text {vis }}^{*}=\rho_{\text {vis }}^{0}+T\left(\mu_{o}, \mu\right) \rho_{\text {vis }} /\left(1-\rho_{\text {vis }} s\right)
$$

where the first term on the right hand side is the atmospheric component, 0 $\rho_{\text {vis, }}$ the path radiance in reflectance units. The second term contains the contribution from the surface with $\mathrm{T}\left(\mu_{\mathrm{o}}, \mu\right)$ the two way transmission through the atmosphere, $\mu_{\mathrm{o}}$ the cosine of the solar zenith angle, $\mu$ the cosine of the satellite view angle, $S$ the spherical albedo from the diffuse reflectance of the atmosphere. When $\rho_{\text {vis }}^{\mathrm{s}}=0$, Equation 3 becomes

$$
\rho_{\text {vis }}^{*}=\rho_{\text {vis }}^{0}
$$


and now without the surface contribution creating confusion in the satellite signal, the aerosol optical thickness can be retrieved directly from $\rho^{*}$ vis. If the path radiance, $\rho_{\text {vis }}^{0}$, can be determined directly, no explicit knowledge of $\rho_{\text {vis }}^{s}$ is necessary.

Figure 8 illustrates the technique for the combined data set of all five flights. The method is based on a scatter plot of visible apparent reflectance, $\rho^{*}$ vis, versus mid-IR apparent reflectance, $\rho^{*} 2.1$. We extrapolate the linear fit of the lower envelope of points to the y-axis where $\rho_{2.1}^{*}=\rho_{2.1}^{s}=0$. If there is no surface reflectance in the mid-IR there should be none in the visible. This is the situation in which Equation 4 holds. Thus the y-intercept of the linear fit is $\rho^{0}$ vis.

The path radiance method was developed for use with Landsat TM data. The method requires homogenous clusters of TM pixels that are treated as the targets. Our targets observed on our flights were chosen by eye for their homogeneity and therefore should resemble the TM pixel clusters used in the analysis. The method was demonstrated with a quarter of a TM scene that contained thousands of targets (Wen et al., 1999). This quantity of data provided sufficient statistics to fit the lower envelope and to estimate the path radiance. Our data set is limited in size. Despite the small size of our data base, we used the data to determine the average errors of the ratio method even when the number of targets fell beneath the acceptable limits of the algorithm. In the same way we will apply the path radiance method to our small data base as a comparison between the two methods.

Despite the poor statistics, we use the surface spectra data collected in Maryland to explore the errors in the path radiance method. Because the data were collected near to the ground there should be no path radiance. The intercept in the scatter plots should be zero. Deviations from a zero intercept 
are error. We apply the path radiance method to the data from each flight and to the total data set of all flights combined. Figure 8 shows the method for the combined data set. The lowest $20 \%$ of the visible reflectance are considered to define the lower envelope and determine the subset of points fitted by the line. The offset in blue is -0.002 and in red -0.01 , both within acceptable limits.

Figure 9 shows all the calculated errors for both the ratio method (from Table 3) and the path radiance method. The errors for the ratio method are determined from the data after all constraints including angular constraints are applied. Three points fall outside of acceptable limits for the ratio method, while four points exceed acceptable error in the path radiance method. The path radiance method does best when there are many targets in the scene distributed over a wide range of $\rho^{*} 2.1$ values and a strong correlation between mid-IR and visible. The path radiance method is not applied unless the correlation coefficient between the visible and mid-IR reflectance is greater than 0.80 . The April blue channel scatter plot is the only flight that does not meet this criterium. The relatively few targets measured in these flights, especially April with its poor correlation, are a poor test for the method. Already the method is self-policing in terms of removing specular reflection targets because it uses only the lower envelope of points to extrapolate to the $y$-axis. Because the path radiance method does not require dark targets, it may increase opportunities to retrieve optical thickness in certain situations. Further testing of the two methods with a larger data base will be necessary to determine the advantages and disadvantages of each.

\subsection{Discussion and Conclusions}


We obtained valuable information on the angular and seasonal variability of surface reflectance using a hand held spectrometer on a light aircraft. In general our data support the hypothesis of a strong correlation between surface reflectance in the visible and mid-IR. We discovered that the correlation is most robust when the view angle is not in the forward scattering direction. As the geometry approaches specular reflection the surface reflectance becomes less spectrally dependent, and the relationships between spectral bands break down.

The ratios between spectral bands follow a seasonal pattern, and are weakly correlated with surface characteristics as quantified by NDVI. The ratios are lowest during the prime growing season when NDVI is highest. During May and July, the months of highest NDVI, the ratios in the red channel are half of what was expected. The errors occur regardless of view angle. The large errors in July at $0.66 \mu \mathrm{m}$ are puzzling. The formulation data set used to derive the original relationships between spectral bands was constructed from July data in similar locations to the area we observed for this study. We would have expected the spectral ratios of the July data in the current data set to most closely resemble the original formulation. However, even the original data set included specific surface types of very low ratios for the 0.66 channel. The current data falls within the scatter of the original measurements.

Using the ratio method to determine surface reflectance in the visible is a viable method to retrieve aerosol optical thickness over land. However, a series of constraints must be imposed in order to capture the darkest targets in the scene. Removing forward scattering pixels will help reduce errors significantly if the view angle approaches specular reflection. The path radiance method proposed by Wen, et al., (1999) avoids determining visible 
surface reflectance explicitly and does not require dark targets. However, further analysis with a larger data base will be necessary to define the necessary constraints on the path radiance method and to evaluate the merits of each technique.

Retrieval of aerosol optical thickness over land continues to be a difficult problem. Understanding the angular dependencies of the retrieval and possibly restricting retrieval near the specular reflection direction will enhance the overall accuracy of the final MODIS aerosol product. Taking advantage of the weak relationship between NDVI and the surface reflectance ratios will need further exploration, as will maximizing the strengths of the ratio method and path radiance method. A comprehensive data set of similar spectrometer data from a low flying airplane was collected over a desert transition zone in Israel. We expect analysis of these data from an alternative biome will help to complete our picture of the effect of NDVI on estimating surface reflectance in the visible, and will eventually lead to an even more accurate MODIS aerosol product.

Acknowledgements. We would like to thank Dr. Guoyong Wen and Dr. Charles Gatebe for reviewing the manuscript and offering very helpful comments. We would also like to thank Beacon Aviation and pilots Mr. Steve Provoncha, Mr. Lloyd Coleman and Mr. Jim Cager for their assistance in collecting the data.

\section{References}


Chu, D.A., Y. J. Kaufman, L. A. Remer and B. N. Holben, Remote sensing of smoke from MODIS airborne simulator during the SCAR-B experiment., $J$. Geophys. Res., 103, 1998.

Deering, D.W., T. F. Eck and B. Banerjee, Characterization of the reflectance anisotropy of three boreal forest canopies in Spring-Summer, Rem. Sens. Environ., 67, 205-229, 1999.

Herman, J.R., P. K. Bhartia, O. Torres, C. Hsu, C. Seftor and E. Celarier, Global distribution of UV-absorbing aerosols from Nimbus 7/TOMS data., J. Geophys. Res., 102, 16911-16922, 1997.

Herman, M., J. L. Deuzé, C. Devaux, P. Goloub, F. M. Bréon and D. Tanré, Remote sensing of aerosols over land surfares including polarization measurements and application to POLDER measurements., J. Geophys. Res., 102, 17,039-17,049, 1997.

Huete, A.R. and C. J. Tucker, Investigation of soil influences in AVHRR vegetation imagery, International Journal of Remote Sensing, ??, ???-???, 1990.

Husar, R.B., L. L. Stowe and J. M. Prospero, Characterization of tropospheric aerosols over the oceans with the NOAA advanced very high resolution radiometer optical thickness operational product, I. Geophys. Res., 102, 1688916910, 1997. 
Kaufman, Y.J. and L. A. Remer, Detection of Forests Using Mid-IR Reflectance: An Application for Aerosol Studies, IEEE Trans. Geoscience Rem. Sens., 32, 672-683, 1994.

Kaufman, Y.J. and C. Sendra, Algorithm for automatic atmospheric corrections to visible and near-IR satellite imagery, International Journal of Remote Sensing, 9, 1357-1381, 1988.

Kaufman, Y.J., D. Tanré, A. Karnieli and L. A. Remer, Re-evaluation of dust radiative forcing using remote measurements of dust aborption of sunlight., J. Geophys. Res., submitted, 1999.

Kaufman, Y.J., D. Tanré, L. A. Remer, E. Vermote, A. Chu and B. N. Holben, Operational remote sensing of tropospheric aerosol over land from EOS moderate resolution imaging spectroradiometer., J. Geophys. Res., 102, 17051$17067,1997$.

Kaufman, Y.J., A. E. Wald, L. A. Remer, B.-C. Gao, R.-R. Li and L. Flynn, The MODIS $2.1 \mu \mathrm{m}$ Channel - Correlation with visible reflectance for use in remote sensing of aerosol., IEEE Trans. Geo, 35, 1286-1298, 1997.

Rondeaux, G. and V. C. Vanderbilt, Specularly modified vegetation indices to estimate photosynthetic activity., Int. J. Rem. Sens., 14, 1815-1823, 1992.

Tanré, D., P. Y. Deschamps, C. Devaux and M. Herman, Estimation of saharan aerosol optical thickness from blurring effects in Thematic Mapper data, J. Geophys. Res., 92, 15955-15964, 1988. 
Tanré, D., Y. J. Kaufman, M. Herman and S. Mattoo, Remote sensing of aerosol properties over oceans using the MODIS/EOS spectral radiances., $J$. Geophys. Res., 102, 16971-16988, 1997.

Tsay, S-C., M. D. King, T. Arnold and J. Y. Li, Airborne spectral measurements of surface anisotropy during SCAR-B, J. Geophys. Res., 103, 31,943-31,953, 1998.

Vanderbilt, V.C. and K. J. De Venecia, Specular, diffuse and polarized imagery of an oat canopy., IEEE Trans. Geoscience and Rem. Sens., 26, 451-462, 1988.

Vermote, E.F., D. Tanré, J. L. Deuzé, M. Herman and J. Morcrette, Second Simulation of the Satellite Signal in the Solar Spectrum: An Overview, IEEE Trans. Geoscience Rem. Sensing. 35, 675-686, 1997

Wen, G., S.-C. Tsay, R. F. Cahalan and L. Oreopoulos, Path radiance technique for retrieving aerosol optical thickness over land., J. Geophys. Res., submitted, 1999.

\section{List of Figures}

Fig 1. Surface reflectance at $0.47 \mu \mathrm{m}$ and $0.66 \mu \mathrm{m}$ as a function of $2.1 \mu \mathrm{m}$ surface reflectance for each of the flights described in Table 1. Linear fits and correlation coefficient are shown. $\mathrm{R}={ }^{*}$ indicates no correlation. 
Figure 2. Surface reflectance for $0.47 \mu \mathrm{m}$ (top) and $0.66 \mu \mathrm{m}$ (bottom) as a function of $2.1 \mu \mathrm{m}$ surface reflectance with all flights plotted on the same axes. The targets viewed from the forward scattering direction are indicated and removed from the plots on the right hand side. Slopes and correlation coefficients are given in Table 2.

Figure 3. Visible surface reflectance as a function of $2.1 \mu \mathrm{m}$ surface reflectance measured over southern Israel. Boxes denote $0.66 \mu \mathrm{m}$ reflectance. Circles denote $0.47 \mu \mathrm{m}$ reflectance. Targets viewed from the forward scattering direction are filled blue. The solar zenith angle was $40^{\circ}$.

Figure 4. Surface reflectance for $0.47 \mu \mathrm{m}$ (top) and $0.66 \mu \mathrm{m}$ (bottom) as a function of $2.1 \mu \mathrm{m}$ surface reflectance with all flights plotted on the same axes. The targets viewed from the forward scattering direction are indicated by blue. Red points are those selected by current constraints and black points are those selected when angular restrictions are added to the current constraints.

Figure 5. Monthly mean ratios between the visible and $2.1 \mu \mathrm{m}$ channel and the Normalized Difference Vegetation Index (NDVI) as a function of month. Shown are different subsets of the data including all targets (solid line), only 
forest targets (dashed line), all wetlands removed (dotted line) and all forward scattering targets removed (solid line with symbols).

Figure 6. Reflectance ratios for the blue (top) and red (bottom) as a function of the Normalized Difference Vegetation Index (NDVI). The linear regression fits and correlation coefficients are given and depicted in the figure by a solid line. The expected values of 0.25 and 0.50 , respectively, are shown by dashed lines. The data represent all targets from all flights with forward scattering targets removed.

Figure 7. Error defined as $\rho_{\lambda}{ }^{\text {meas }}-\rho_{\lambda}{ }^{\text {calc }}$ for $\lambda=0.47 \mu \mathrm{m}$ and $0.66 \mu \mathrm{m}$ as a function of month for different combinations of pixel selection. No constraints indicates all pixels were included. Dark targets indicates that only targets with $\rho_{2.1}<0.15$ are included. Current restraints indicates that both the dark target criterion and a filter that eliminates the $10 \%$ darkest targets and the $60 \%$ brightest targets are imposed. "No forw" indicates that all forward scattering targets were removed before filter was applied. The last "month" plotted is actually a combination of all the targets from all five flights.

Figure 8. Path radiance method (Wen et al., 1999) applied to the total data set for the $0.47 \mu \mathrm{m}$ channel (top) and the $0.66 \mu \mathrm{m}$ channel (bottom). Because the data are taken near the ground there should be no path radiance and the yintercept of the fit to the lower envelope of points should be zero. Offsets 
deviating from zero define the method's error. The lower envelope is defined by the lowest $20 \%$ of the visible reflectance.

Figure 9. Average errors resulting from applying the two surface retrieval methods for each of the monthly data sets and to the total data set for both channels plotted as a function of mean reflectance at $2.1 \mu \mathrm{m}$. The ratio method errors are the difference of the measured and calculated values of surface reflectance after all three constraints are applied to the data and are denoted by open symbols. The path radiance errors are the offset of the line fit to the lower envelope of points for each of the data sets and are denoted by filled symbols. The ratio method is a dark target method, and therefore the targets remaining after constraints are applied have lower $\rho_{2.1 \text { mean }}$ than the targets used in the path radiance method, which uses all targets, bright or dark. 
Table 1 Dates and times of each flight, whether angular information was recorded, the aerosol optical thickness at $670 \mathrm{~nm}\left(\tau_{670}\right)$ during the flight, the solar zenith angle $\left(\theta_{0}\right)$ at the time of the flight and the mean Normalized Difference Vegetation Index (NDVI). Flights are arranged in seasonal progression, but span two years.

\begin{tabular}{|l|l|l|l|l|l|}
\hline Date & $\begin{array}{l}\text { Time of } \\
\text { Takeoff }\end{array}$ & $\begin{array}{l}\text { Angles } \\
\text { Noted }\end{array}$ & $\tau_{670}$ & $\theta_{\mathrm{o}}$ & NDVI \\
\hline March 12, 1997 & $10: 10$ EST & Yes & 0.10 & $46^{\circ}$ & 0.45 \\
\hline April 22, 1996 & $8: 15$ EST & Yes & 0.10 & $51^{\circ}$ & 0.53 \\
\hline May 22, 1996 & $12: 35$ EST & No & 0.20 & $23^{\circ}$ & 0.82 \\
\hline July 30, 1997 & $13: 05$ EST & No & 0.05 & $32^{\circ}$ & 0.81 \\
\hline October 16, 1996 & $12: 45$ EST & Yes & 0.15 & $51^{\circ}$ & 0.56 \\
\hline
\end{tabular}


Table 2 Ratios of $\rho_{0.47} / \rho_{2.1}$ (top) and $\rho_{0.67} / \rho_{2.1}$ (bottom) and correlation coefficients for all targets, targets excluding marshes, swamps and estuaries, only forest targets, and targets of all surface types but no forward scattering views.

\begin{tabular}{|c|c|c|c|c|c|c|c|c|}
\hline \multirow[t]{2}{*}{ Month } & \multicolumn{2}{|c|}{ All Targets } & \multicolumn{2}{|c|}{ No Marsh } & \multicolumn{2}{|c|}{ Only Forest } & \multicolumn{2}{|c|}{ No Forward } \\
\hline & ratio & $\mathbf{R}$ & ratio & $\mathrm{R}$ & ratio & $\mathrm{R}$ & ratio & $\mathbf{R}$ \\
\hline March & 0.29 & 0.95 & 0.29 & 0.95 & 0.30 & 0.69 & 0.29 & 0.96 \\
\hline April & 0.41 & - & 0.41 & - & 0.43 & - & 0.36 & 0.83 \\
\hline May & 0.21 & 0.64 & 0.19 & 0.85 & 0.16 & 0.52 & \multicolumn{2}{|c|}{ No angles } \\
\hline July & 0.21 & 0.95 & 0.21 & 0.95 & 0.20 & 0.71 & \multicolumn{2}{|c|}{ No angles } \\
\hline October & 0.34 & - & 0.32 & - & 0.44 & - & 0.29 & 0.79 \\
\hline
\end{tabular}

\begin{tabular}{|l|l|l|l|l|l|l|l|l|}
\hline Month & \multicolumn{2}{|l|}{ All Targets } & \multicolumn{2}{l|}{ No Marsh } & \multicolumn{2}{l|}{ Only Forest } & \multicolumn{2}{l|}{ No Forward } \\
\hline & ratio & $\mathrm{R}$ & ratio & $\mathrm{R}$ & ratio & $\mathrm{R}$ & ratio & $\mathrm{R}$ \\
\hline \hline March & 0.46 & 0.98 & 0.46 & 0.98 & 0.42 & 0.97 & 0.46 & 0.98 \\
\hline April & 0.50 & 0.51 & 0.50 & 0.51 & 0.51 & - & 0.47 & 0.90 \\
\hline May & 0.39 & 0.90 & 0.35 & 0.91 & 0.23 & 0.71 & \multicolumn{2}{|l|}{ No angles } \\
\hline July & 0.38 & 0.97 & 0.39 & 0.97 & 0.28 & 0.93 & \multicolumn{2}{|l|}{ No angles } \\
\hline October & 0.53 & 0.80 & 0.51 & 0.88 & 0.56 & 0.46 & 0.50 & 0.96 \\
\hline
\end{tabular}


Table 3. Error analysis by month and for total combined data set.

\begin{tabular}{|c|c|c|c|c|c|c|c|}
\hline & & March & April & May & July & October & Total \\
\hline \multirow[t]{3}{*}{$\begin{array}{l}\text { No } \\
\text { constraints }\end{array}$} & $\mathrm{N}$ & 27 & 15 & 18 & 25 & 40 & 125 \\
\hline & error 0.47 & 0.011 & 0.034 & -0.003 & -0.005 & 0.024 & 0.013 \\
\hline & error 0.66 & -0.010 & 0.005 & -0.010 & -0.023 & 0.011 & -0.004 \\
\hline \multirow[t]{3}{*}{$\begin{array}{l}\text { Dark targets. } \\
\text { ( } 0.15 \text { cutoff }\end{array}$} & $\mathrm{N}$ & 13 & 5 & 16 & 16 & 18 & 68 \\
\hline & error 0.47 & 0.011 & 0.045 & -0.002 & -0.003 & 0.030 & 0.012 \\
\hline & error 0.66 & -0.009 & 0.018 & -0.010 & -0.018 & 0.019 & -0.002 \\
\hline \multirow{3}{*}{$\begin{array}{l}\text { Current } \\
\text { constraints } \\
(0.15 \quad \text { cutoff } \\
\text { and } 10-40 \% \\
\text { filter) }\end{array}$} & $\mathrm{N}$ & 4 & 2 & 5 & 5 & 6 & 20 \\
\hline & error 0.47 & 0.007 & 0.021 & -0.007 & -0.007 & 0.008 & -0.002 \\
\hline & error 0.66 & -0.006 & 0.007 & -0.012 & -0.016 & 0.013 & -0.015 \\
\hline \multirow{3}{*}{$\begin{array}{l}\text { Current } \\
\text { constraints } \\
\text { and angular } \\
\text { restrictions }\end{array}$} & $\mathrm{N}$ & 2 & 0 & \multirow{2}{*}{\multicolumn{2}{|c|}{$\begin{array}{l}\text { Angles not noted } \\
\text { Same as current }\end{array}$}} & 4 & 16 \\
\hline & error 0.47 & 0.002 & & & & 0.006 & -0.006 \\
\hline & error 0.66 & -0.010 & & constra & & 0.009 & -0.015 \\
\hline \multirow{3}{*}{$\begin{array}{l}0.15 \text { cutoff } \\
\text { and angular } \\
\text { restrictions, } \\
\text { but no 10- } \\
40 \% \text { filter }\end{array}$} & $\mathrm{N}$ & 6 & 1 & 16 & 16 & 15 & 53 \\
\hline & error 0.47 & 0.010 & 0.032 & -0.002 & -0.003 & 0.023 & 0.006 \\
\hline & error 0.66 & -0.009 & 0.005 & -0.010 & -0.018 & 0.014 & -0.005 \\
\hline \multirow{3}{*}{$\begin{array}{l}10-40 \% \text { filter } \\
\text { and angular } \\
\text { restrictions } \\
\text { but no cutoff }\end{array}$} & $\mathrm{N}$ & 5 & 2 & 5 & 8 & 8 & 28 \\
\hline & error 0.47 & 0.012 & 0.025 & -0.007 & -0.006 & 0.006 & -0.001 \\
\hline & error 0.66 & -0.009 & -0.005 & -0.012 & -0.018 & 0.007 & -.010 \\
\hline
\end{tabular}




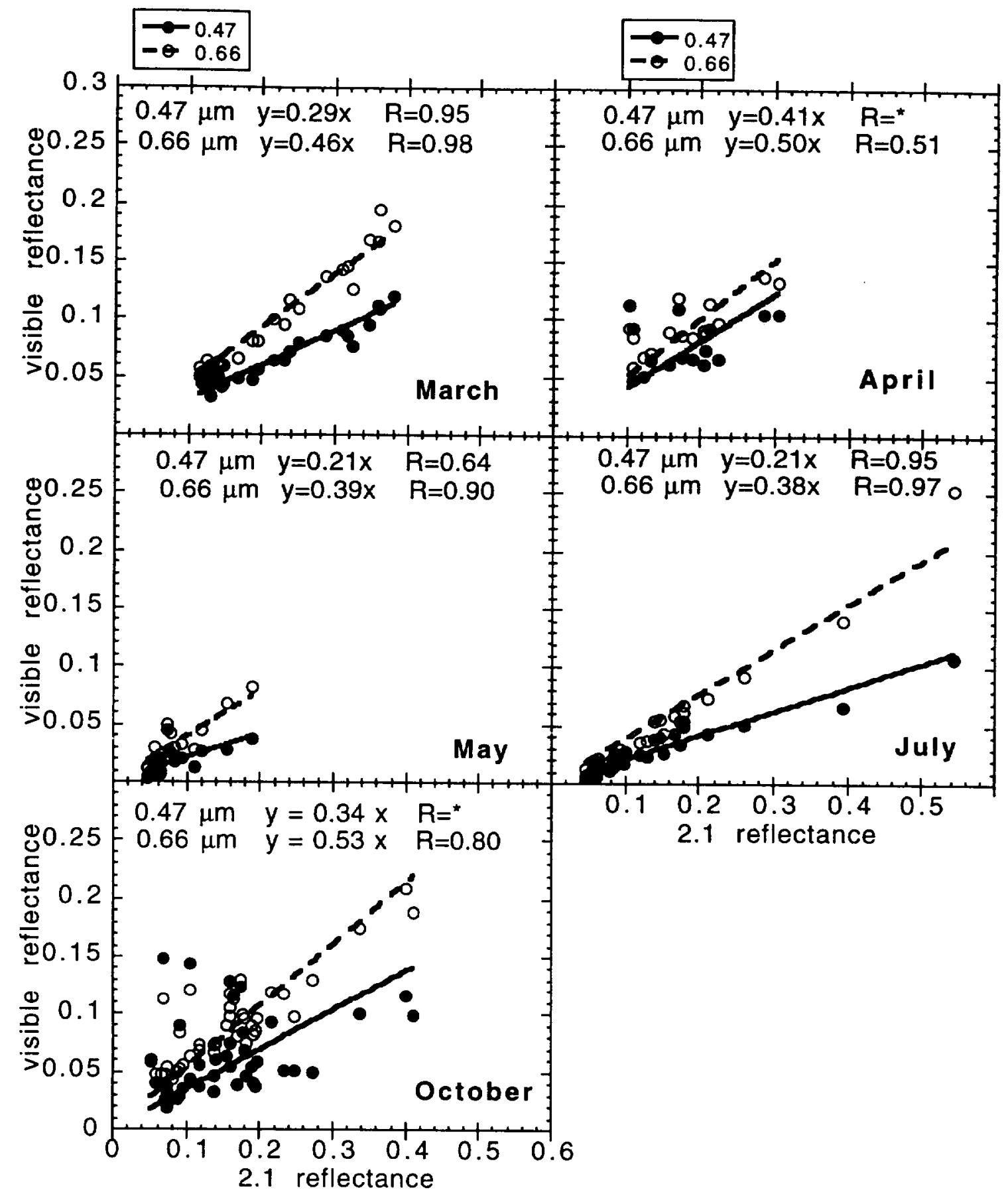

Fig 1. Surface reflectance at $0.47 \mu \mathrm{m}$ and $0.66 \mu \mathrm{m}$ as a function of $2.1 \mu \mathrm{m}$ surface reflectance for each of the flights described in Table 1. Linear fits and correlation coefficient are shown. $\mathrm{R}=*$ indicates no correlation. 


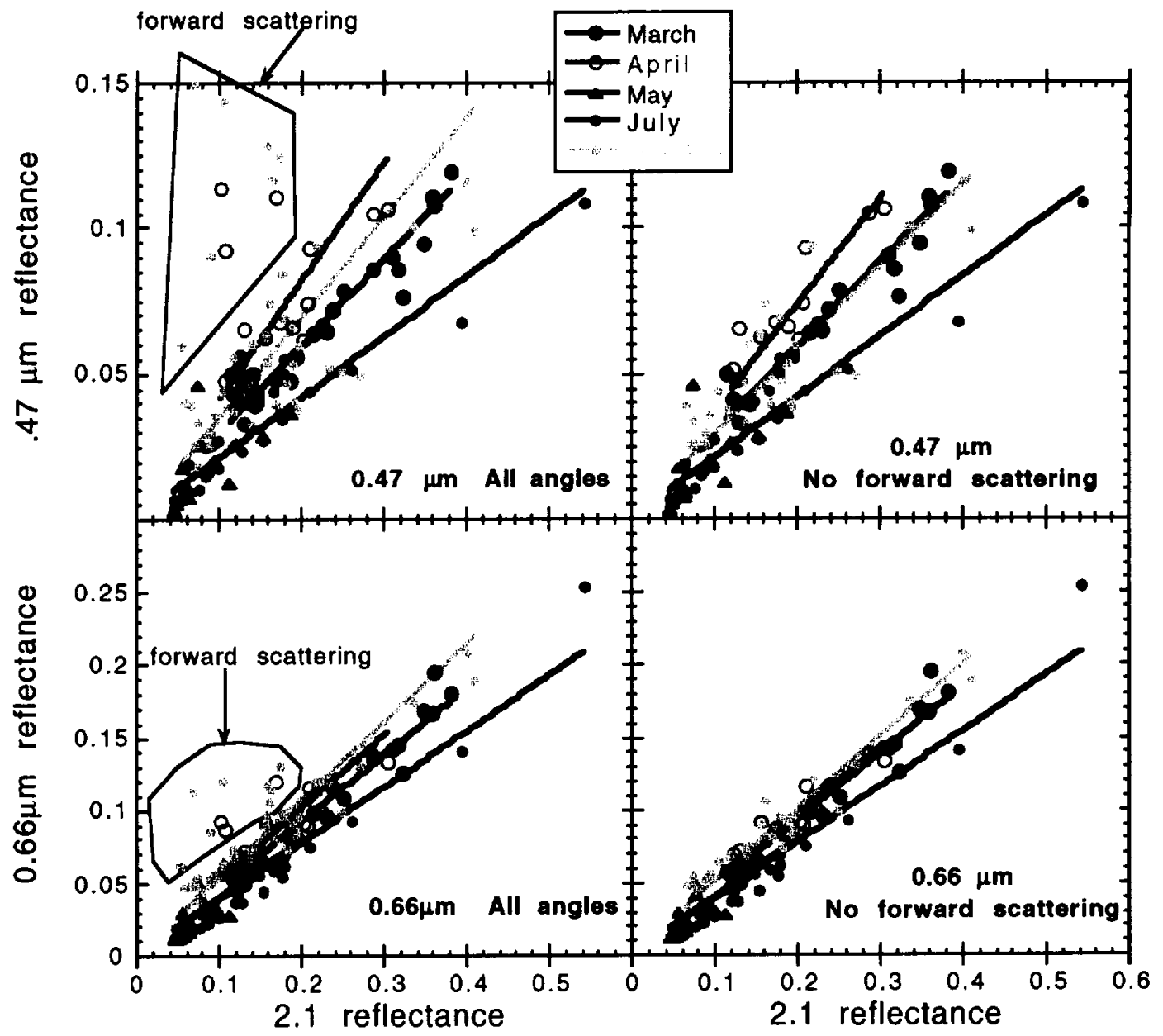

Figure 2. Surface reflectance for $0.47 \mu \mathrm{m}$ (top) and $0.66 \mu \mathrm{m}$ (bottom) as a function of $2.1 \mu \mathrm{m}$ surface reflectance with all flights plotted on the same axes. The targets viewed from the forward scattering direction are indicated and removed from the plots on the right hand side. Slopes and correlation coefficients are given in Table 2. 


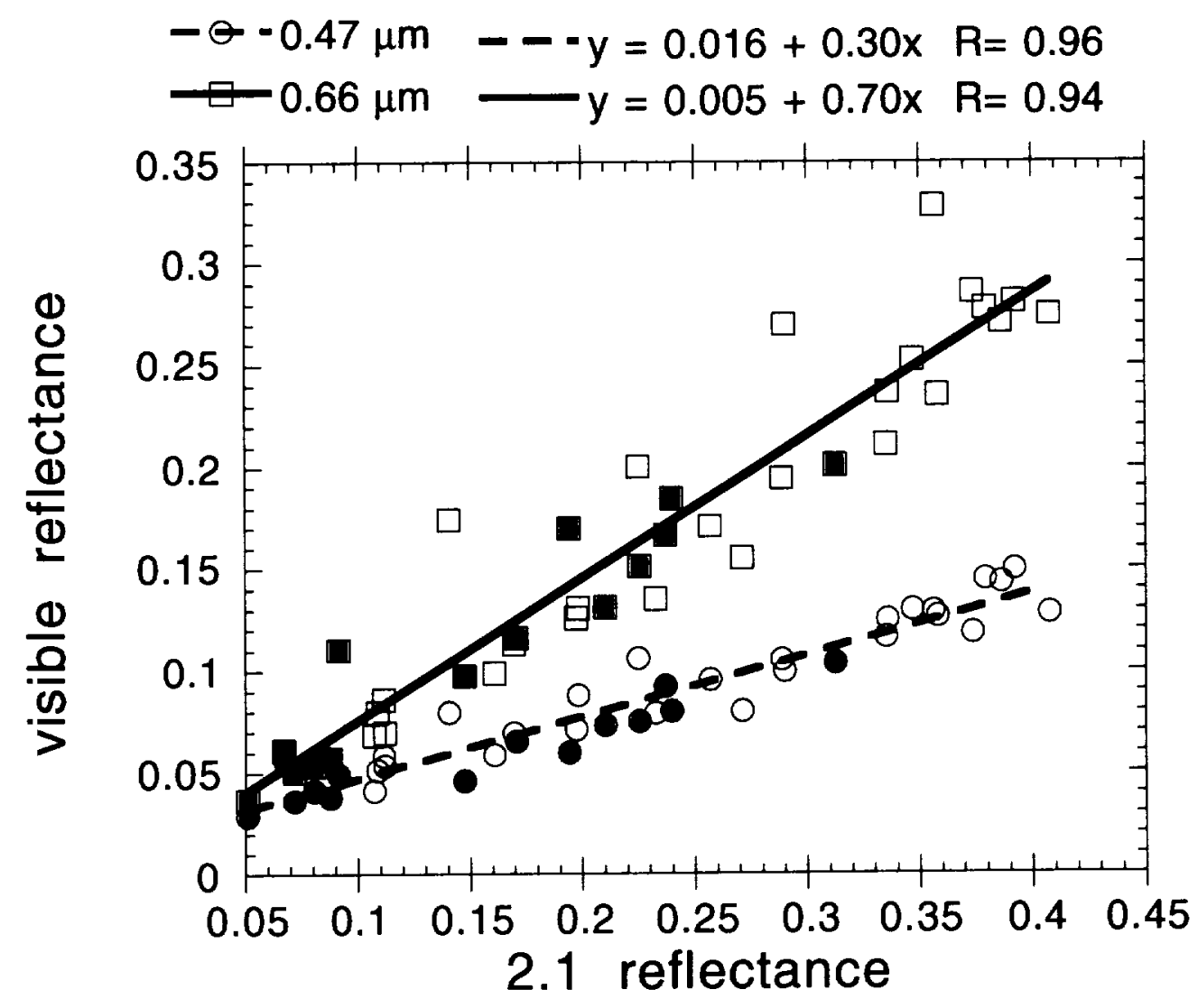

Figure 3. Visible surface reflectance as a function of $2.1 \mu \mathrm{m}$ surface reflectance measured over southern Israel. Boxes denote $0.66 \mu \mathrm{m}$ reflectance. Circles denote $0.47 \mu \mathrm{m}$ reflectance. Targets view from the forward scattering direction are filled blue. The solar zenith angle was $40^{\circ}$. 


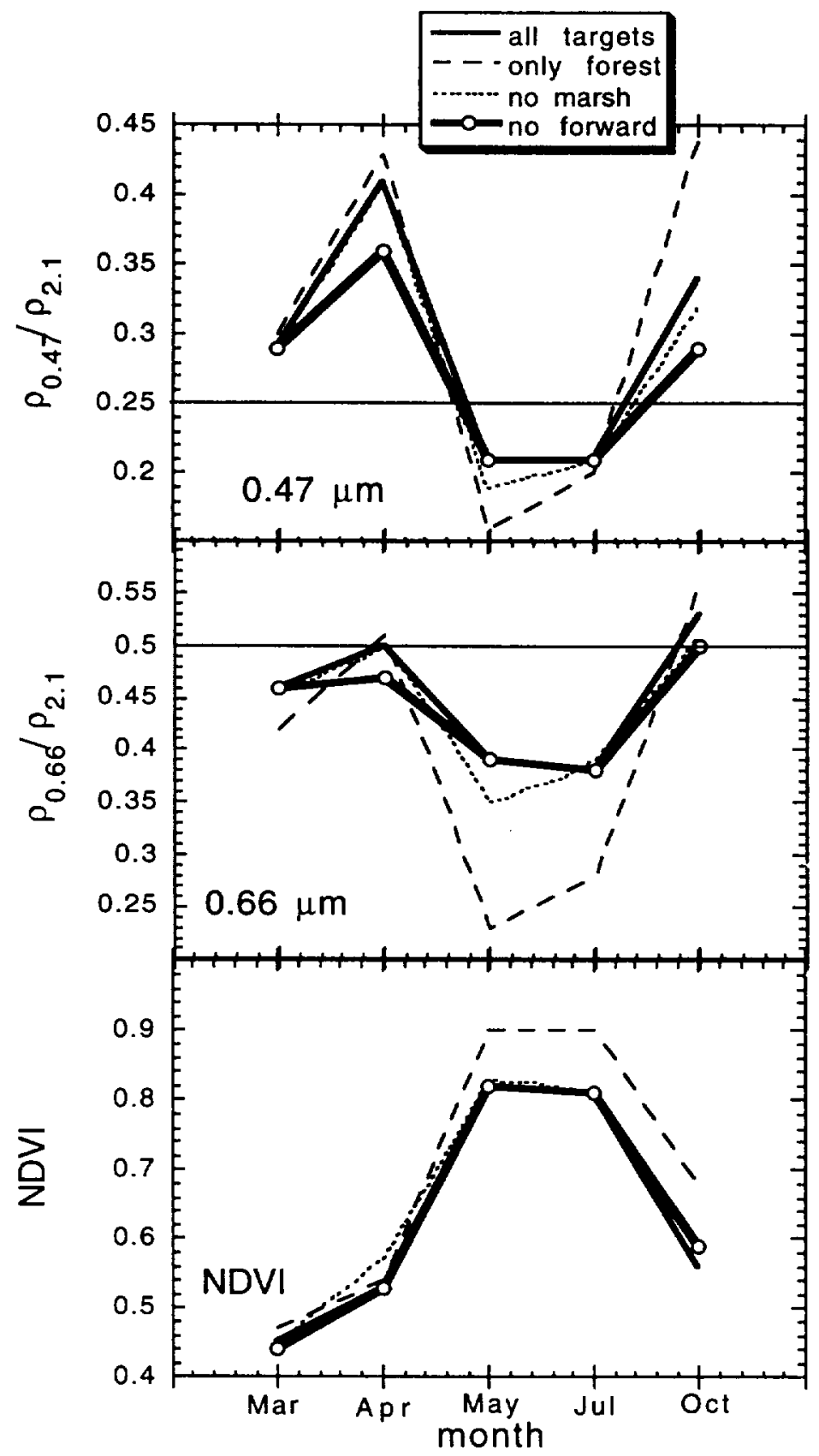

Figure 5. Monthly mean ratios between the visible and $2.1 \mu \mathrm{m}$ channel and the Normalized Difference Vegetation Index (NDVI) as a function of month. Shown are different subsets of the data including all targets, only forest targets, all welands removed and all forward scattering targets removed. 

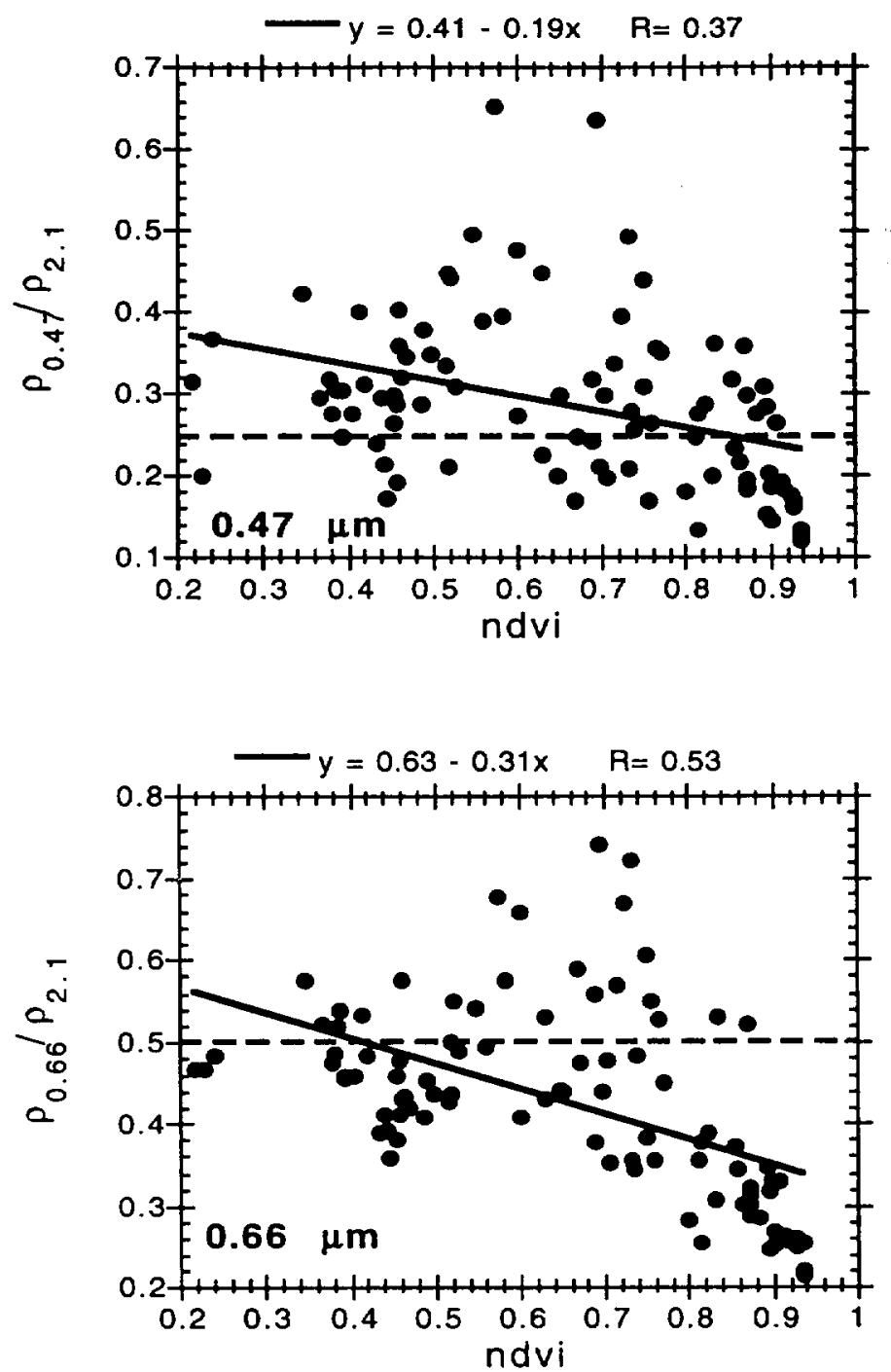

Figure 6. Reflectance ratios for the blue (top) and red (bottom) as a function of NDVI. The linear regression fits and correlation coefficients are given and depicted in the figure by a solid line. The expected values of 0.25 and 0.50 , respectively, are shown by dashed lines. The data represent all targets from all flights with forward scattering targets removed. 


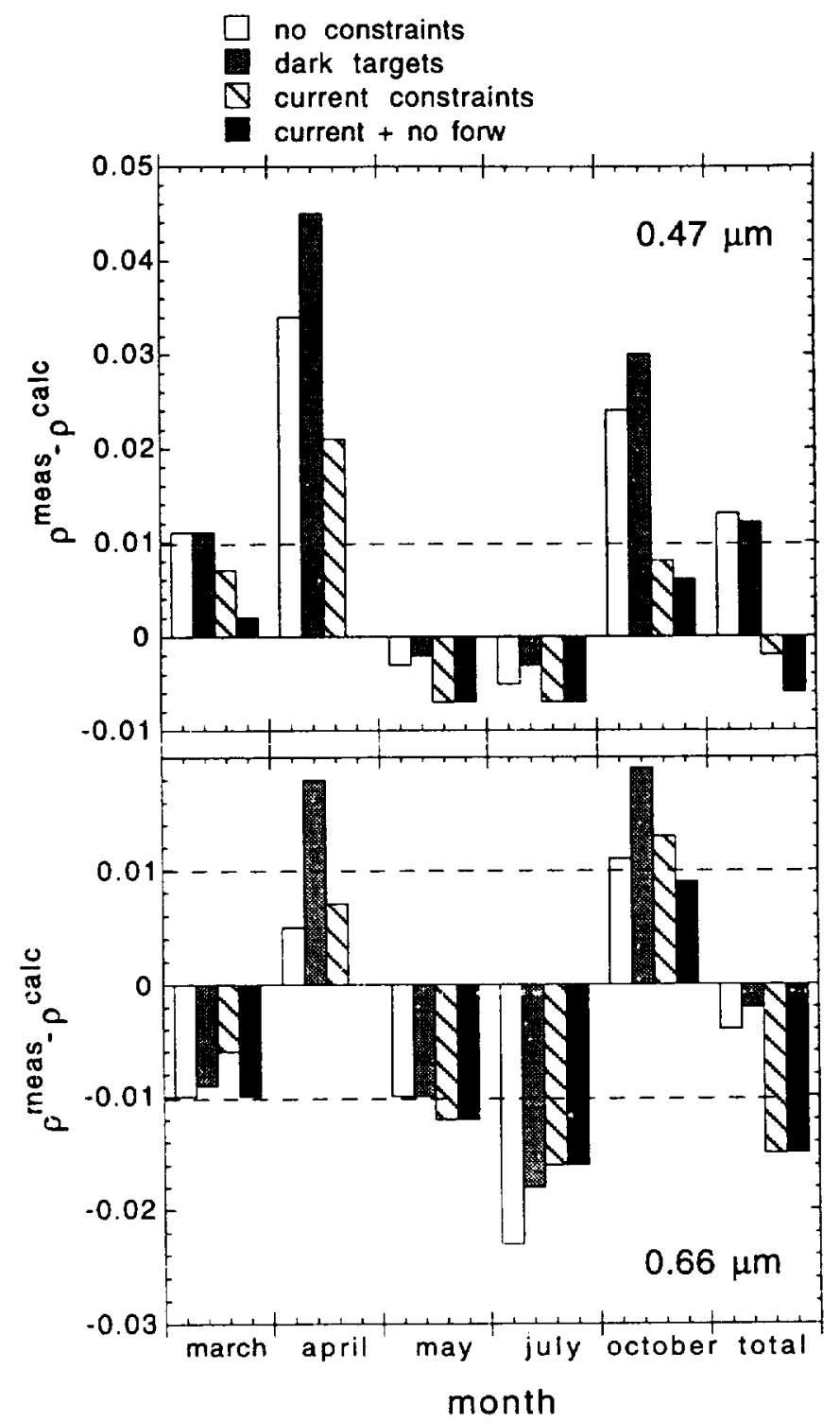

Figure 7. Error defined as $\rho_{\lambda}{ }^{\text {meas }}-\rho_{\lambda}{ }^{\text {calc }}$ for $\lambda=0.47 \mu \mathrm{m}$ and $0.66 \mu \mathrm{m}$ as a function of month for different combinations of pixel selection. No constraints indicates all pixels were included. Dark targets indicates that only targets with $\rho_{2.1}<0.15$ are included. Current constraints indicates that both the dark target criterion and a filter that eliminates the $10 \%$ darkest targets and the $60 \%$ brightest targets are imposed. No forw indicates that all forward scattering targets were removed before filter was applied. The last "month" plotted is actually a conglomeration of all the targets from all five flights. 

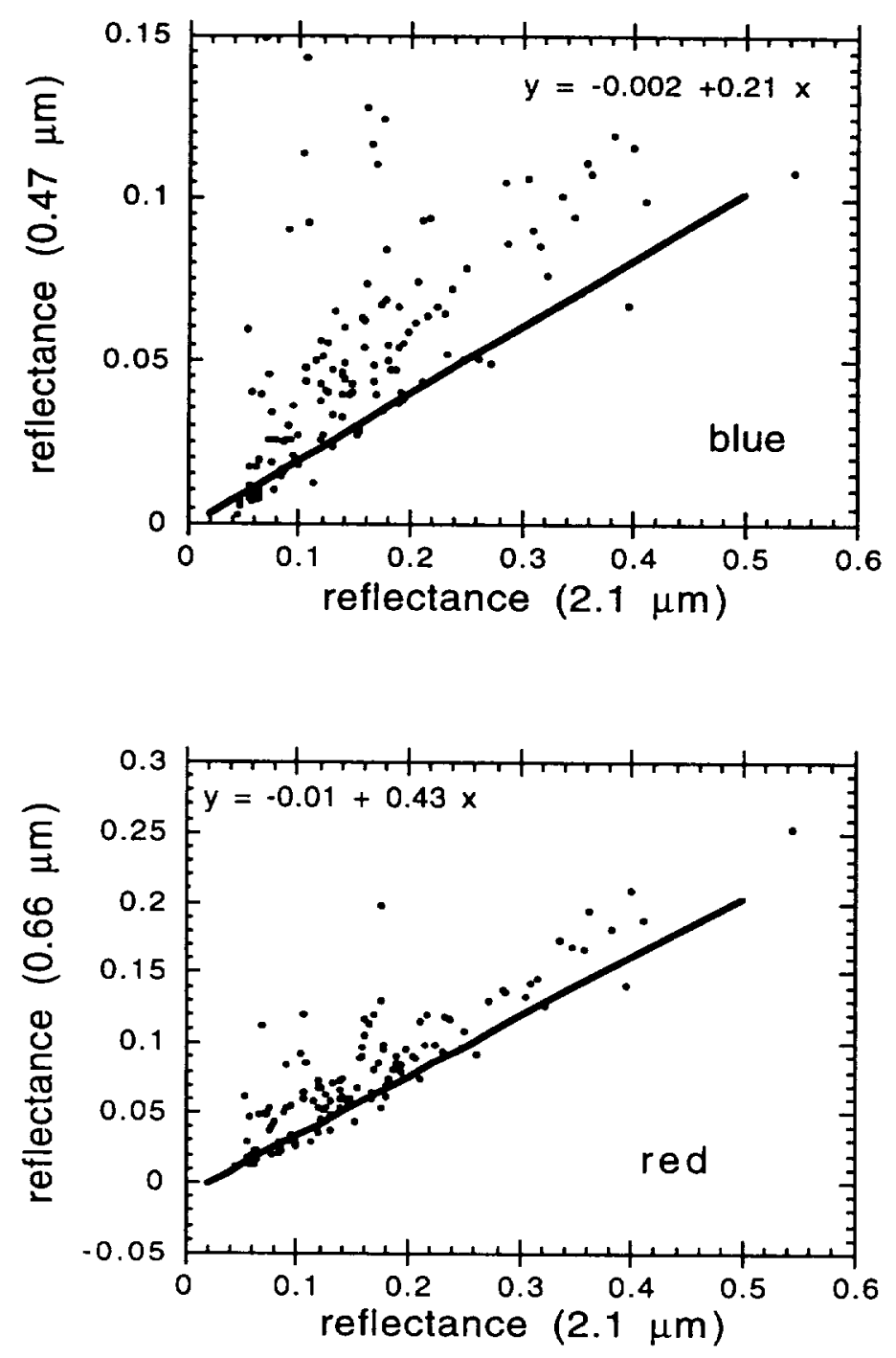

Figure 8. Path radiance method applied to the total data set for the $0.47 \mu \mathrm{m}$ channel (top) and the $0.66 \mu \mathrm{m}$ channel (bottom). Because the data are taken near the ground there should be no path radiance and the $y$-intercept of the fit to the lower envelope of points should be zero. Offsets deviating from zero define the method's error. The lower envelope is defined by the lowest $20 \%$ of the visible reflectance. 


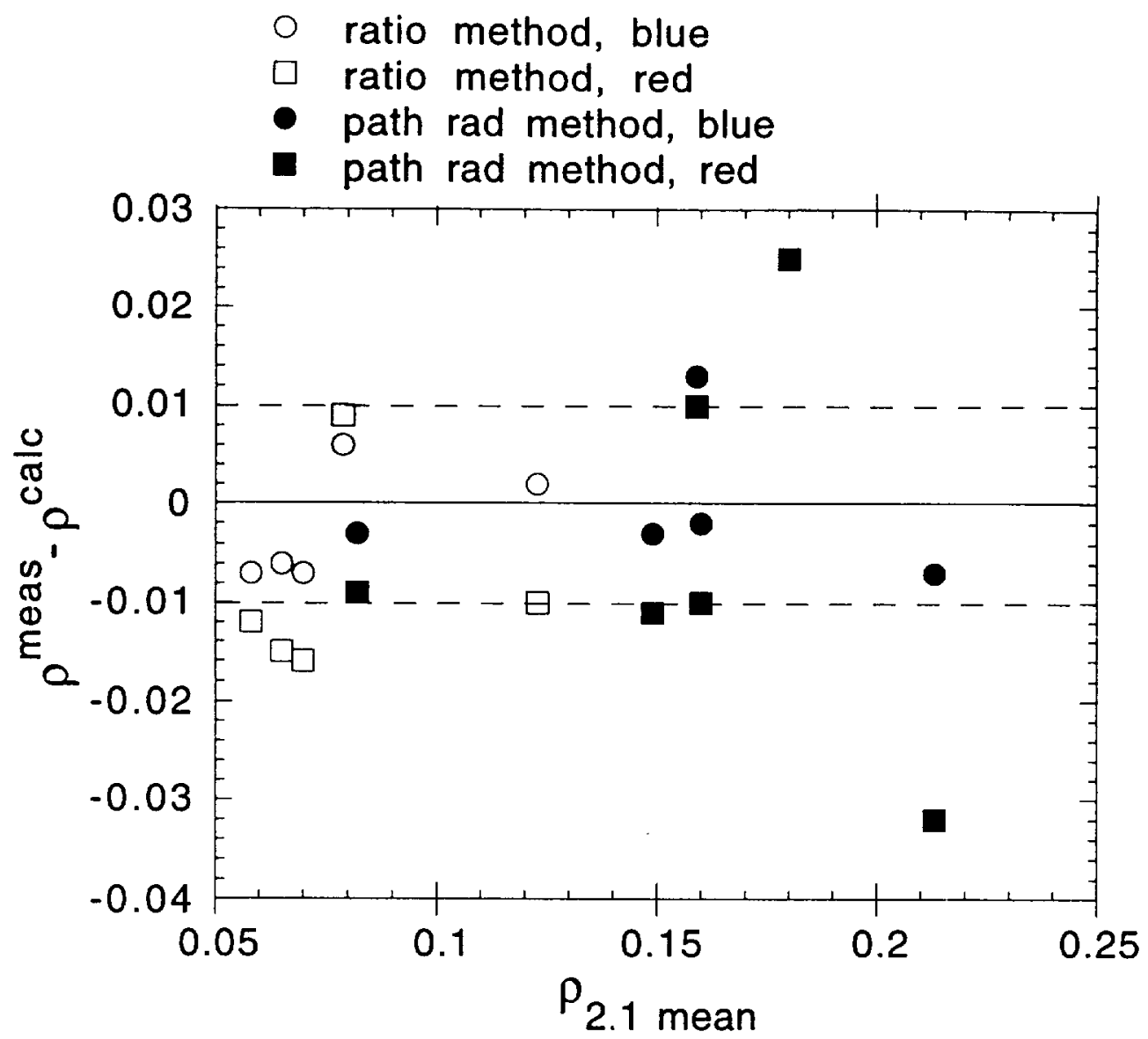

Figure 9. Average errors resulting from applying the two surface retrieval methods for each of the flights and to the total data set for both channels plotted as a function of mean reflectance at $2.1 \mu \mathrm{m}$. The ratio method errors are the difference of the measured and calculated values of surface reflectance after all three constraints are applied to the data and are denoted by open symbols. The path radiance errors are the offset of the line fit to the lower envelope of points for each of the data sets and are denoted by filled symbols. The ratio method is a dark target method, and therefore the targets remaining after constraints are applied have lower $\rho_{2.1 \text { mean }}$ than the targets used in the path radiance method, which uses all targets, bright or dark. 